Research Article

\title{
Effect of Rotational Speed on Performance of Mixed Flow Pump as Turbine
}

\author{
Jun Chen, ${ }^{1}$ Tao Dai $\mathbb{D}^{2}{ }^{2}$ and Qing Yang $\mathbb{D D}^{1}$ \\ ${ }^{1}$ School of Computer Engineering, Nanjing Institute of Technology, Nanjing, China \\ ${ }^{2}$ Research Center of Fluid Machinery Engineering and Technology, Jiangsu University, Zhenjiang, China \\ Correspondence should be addressed to Qing Yang; yangq@njit.edu.cn
}

Received 16 November 2020; Revised 9 December 2020; Accepted 29 January 2021; Published 10 February 2021

Academic Editor: Denise-Penelope Kontoni

Copyright (c) 2021 Jun Chen et al. This is an open access article distributed under the Creative Commons Attribution License, which permits unrestricted use, distribution, and reproduction in any medium, provided the original work is properly cited.

In order to research the flow characteristics of mixed flow pump as turbine (MF-PAT) with different rotation speeds, a hydraulic model of mixed flow pump was established based on a pumped storage power station. The $k-\varepsilon$ turbulent model was used to simulate internal flow fields with three rotation speeds by SMPLEC algorithms. Subsequently, theoretical calculations and experiments were carried out to verify the precision of numerical simulation. The results showed that the rotation speed of MF-PAT has a significant impact on its performance. Both numerical calculations and experimental test presented that all efficiency curves consist of ascending and descending stages, while the shaft power and head increase nonlinearly from 1000 to $2000 \mathrm{r} / \mathrm{min}$. When the MF-PAT deviates from the rated environment, increase in speed is positive to the energy recovery efficiency in the high water period and negative in the dry season. This work could provide a reference for further study of MF-PAT.

\section{Introduction}

Global warming is the main factor that endangers the balance of natural ecosystem and threatens the survival of human beings, which has launched a more intensive use of renewable energy on a global scale [1]. According to International Renewable Energy Agency (IRENA) survey of renewable energy sources worldwide, total installed hydropower capacity (excluding pumped storage) was 1,307,994 MW until the end of 2019 [2]. Mixed flow pumps have several advantages for pico-hydropower plant compared to purpose-made turbines. Mixed flow pumps have availability in a large number of standard sizes for a wide range of heads and flows, short delivery time, long life span, and easy installation and availability of spare parts such as seals [3-5]. The pump, as a reversible rotating machine, converts the liquid pressure energy into the mechanical energy of the impeller to drive the generator when it is running in reverse, and this operation is called pump as turbine (PAT). It is widely used in industrial residual pressure recovery system and remote rural where electricity is scarce. With comprehensive hydraulic considerations, mixed flow pump as turbine (MF-PAT) is recommended by many researchers in the low-capacity range (up to $100 \mathrm{~kW}$ ) of hydropower development [6-8]. This type of PAT is suitable for the low head and large discharge environment.

MF-PAT works at different loads for several months in a year due to the change in hydropower potential. The application range of PAT can be widened if its maximum efficiency can be improved $[9,10]$. Many studies have investigated the energy efficiency ratio and flow law of PAT under the constant speed. At present, scholars have focused on three aspects of PAT: pump selection, hydraulic characteristics, and performance optimization [11-16].

The power generation of micro-hydropower stations is subject to natural factors, and the characteristics of large flow and low head are more obvious. Under this work condition, the MF-PAT is more suitable $[17,18]$. With the development of frequency conversion technology, which could change the turbine speed to adjust the operating conditions, the long-term efficient operation of the turbine can be realized $[19,20]$. Nevertheless, rotational speed on performance of MF-PAT has been rarely reported and its effect and mechanism are unclear nowadays. Therefore, it is 
necessary to study and analyze the performance of MFPAT with variable speed conditions.

In this paper, based on a model of MF-PAT with a specific speed of 288, numerical analysis and experiments are used to analyze the external characteristics of the MF-PAT from 1000 to $2000 \mathrm{r} / \mathrm{min}$. Firstly, the internal flow field and its distribution are analyzed. Then, a test rig of open hydraulic turbine is used to test the external characteristic performance of MF-PAT to verify the numerical calculation, and the results are compared and analyzed. Finally, according to the influence of rotational speeds on MF-PAT, a cost-effective operation scheme is proposed. This study could provide a reference for the MF-PAT to recover energy.

\section{Model of Mixed Flow Pump as Turbine}

The parameters of model of MF-PAT in the paper are as follows: the rated head is $5 \mathrm{~m}$, the rated rotating speed is $1500 \mathrm{r} / \mathrm{min}$, and the rated flow rate is $120 \mathrm{~m}^{3} / \mathrm{h}$; Figure 1 shows the computational domain of MF-PAT, which includes draft tube, front cavity, impeller, back cavity, and volute. The main parameters of the MF-PAT are listed in Table 1.

\section{Numerical Investigation}

3.1. Mesh Arrangement. As shown in Figure 2, structured hexahedral meshes were generated for the entire computational domain in ICEM. The "O" topology method and high density of the elements were applied on the wall boundary layer to improve the accuracy of numerical calculation. The average value of $y+$ near the boundary wall is around 15. After grid independence verification, the efficiency varied by less than $0.5 \%$ when the grid numbers are more than $1.2 \times 10^{6}$.

To study the effect of the number of meshes on the numerical results, five different sizes of grids were selected to calculate the MF-PAT, and the number of grids was controlled by varying the global size and the number of nodes. Calculation results under design conditions $\left(120 \mathrm{~m}^{3} / \mathrm{h}\right.$, $1500 \mathrm{rpm}$ ) are analyzed with head and efficiency as comparison factors, as shown in Figure 3. It can be seen that as the number of meshes increases, the trend of efficiency and head decreases. When the number of meshes is greater than 1.2 million, it can be approximated that there is no change, and the numerical simulation results are independent of the number of meshes in this interval. The number of meshes in the computational model in this paper is greater than 1.2 million.

3.2. Numerical Method. Ansys-CFX was used to calculate the flow field of the MF-PAT. The $k-\varepsilon$ turbulence model was used in steady simulation. The boundary conditions were set as follows: the total pressure at the inlet, the mass flow at the outlet, and the wall function is defined as scalable, and the wall roughness is set to $0.5 \mathrm{~mm}$ [21-23]. Moreover, the root mean square (RMS) residual was set as $1 \times 10^{-5}$ for the convergent criterion. The frozen rotor and transient rotor stator methods were used for the coupling interface of the

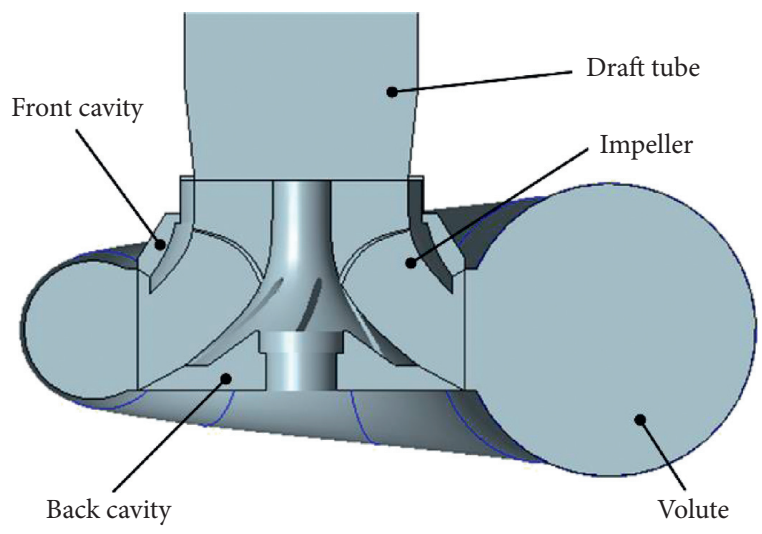

Figure 1: 3D model of the internal flow field of MF-PAT.

TABLE 1: Main geometric parameters of impeller and volute of turbine.

\begin{tabular}{lcc}
\hline Component & Parameter & Value \\
\hline \multirow{4}{*}{ Impeller } & Hub diameter $D_{h}(\mathrm{~mm})$ & 25 \\
& Inlet diameter $D_{1}(\mathrm{~mm})$ & 115 \\
& Outlet diameter $D_{2}(\mathrm{~mm})$ & 92 \\
& Inlet width $b_{2}(\mathrm{~mm})$ & 34 \\
& Blade wrap angle $\varphi\left(^{\circ}\right)$ & 45 \\
\hline \multirow{4}{*}{ Volute } & Base circle diameter $D_{3}(\mathrm{~mm})$ & 140 \\
& Inlet diameter $D_{5}(\mathrm{~mm})$ & 150 \\
& Outlet width $b_{3}(\mathrm{~mm})$ & 56 \\
& Section shape & Circle \\
\hline
\end{tabular}

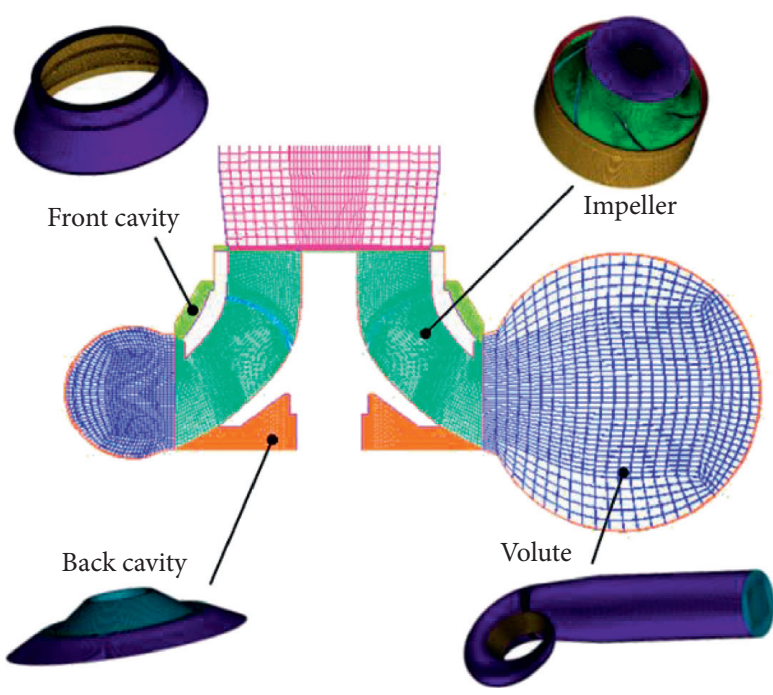

FIgURE 2: Mesh of turbine's computational domain.

rotational and stationary domains in steady simulations. In particular, there were three rotational speeds with 1000 , 1500 , and $2000 \mathrm{r} / \mathrm{min}$ of MF-PAT considered in this paper.

3.3. Numerical Results. Figure 4 shows the pressure distributions at $z=0$ plane. It can be found that the pressure at the inlet of the impeller gradually reduces at high-efficiency points from 1000 to $2000 \mathrm{r} / \mathrm{min}$. The increase in flow could 


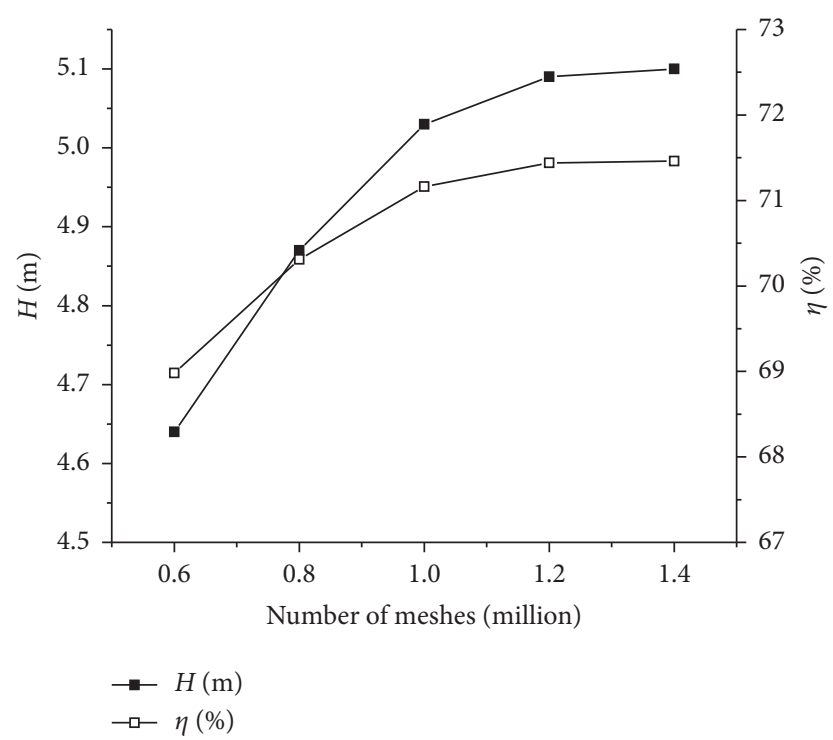

Figure 3: Efficiency, head, and meshes number curve.

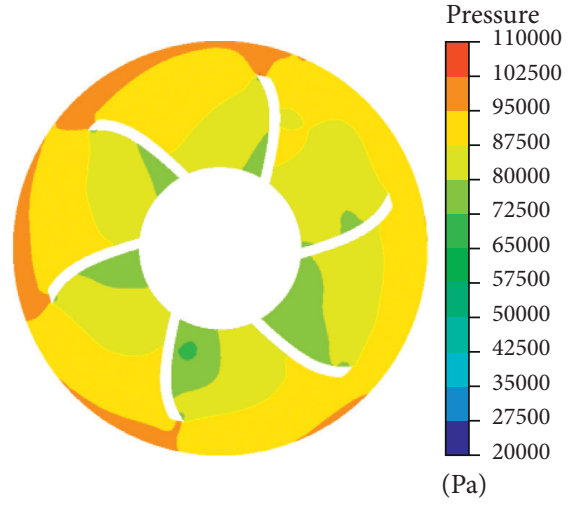

(a)

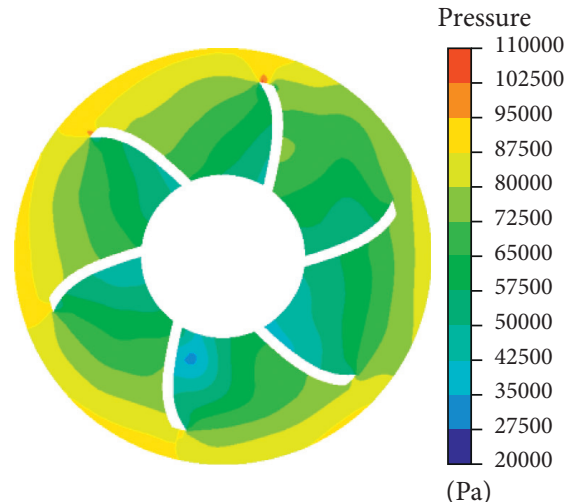

(b)

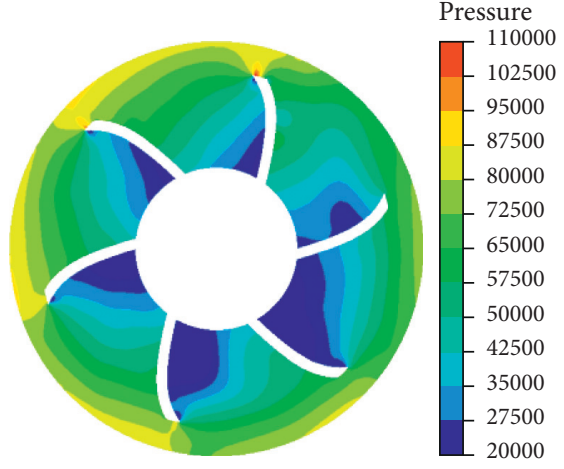

$(\mathrm{Pa})$

(c)

Figure 4: Pressure distributions of $z=0$ plane: (a) $1000 \mathrm{r} / \mathrm{min}\left(80 \mathrm{~m}^{3} / \mathrm{h}\right)$; (b) $1500 \mathrm{r} / \mathrm{min}\left(120 \mathrm{~m}^{3} / \mathrm{h}\right)$; (c) $2000 \mathrm{r} / \mathrm{min}\left(160 \mathrm{~m}^{3} / \mathrm{h}\right)$.

lead to the rise of circulation velocity and the decline in pressure at the outlet of the volute. From the inlet position of the impeller to the outlet position, the liquid pressure gradually decreases. Also, with the increase in rotation speed and flow rate, the pressure gradient increases on both sides of the impeller blades, especially at the tail of the blade. And, pressure on the suction pressure face is smaller than that on the normal surface. The phenomenon may be caused by the increase in force pushing on the impeller and the rise of the pressure recovery capability. Due to the asymmetry of the volute structure, pressure distribution in the impeller is not uniform, and it is more turbulent in the area near the volute tongue.

Figure 5 shows the velocity streamlines of impeller at $z=0$ plane. The increase in rotation speed causes the increase in flow speed. However, the shape of the streamlines has no obvious change. The vortexes distribute in small passage of the impeller and have a gradually increasing strength. These vortexes have a notable influence on the efficiency of MF-PAT.
Figure 6 shows the performance curves of MF-PAT with different rotation speeds obtained by CFD. The performance curves show a similar trend at different speeds. The efficiency curves can be divided into an ascending stage and descending stage. Notably, there are significant effects of rotational speeds on MF-PAT's performance. Under the same flow rates, the required head of MF-PAT and the output shaft power increase with the speed. Concurrently, the best efficiency point (BEP) obviously shift to the large-flow conditions. The scope of the high-efficiency zone has been expanded, but the highest efficiency value has not increased significantly. Table 2 lists the parameters of BEP by CFD with different rotation speeds.

3.4. Theoretical Analysis. On continuing further analysis, the circumferential velocity at the inlet $u_{1}$ is related and proportional to the rotational speed, as shown in the following equation: 


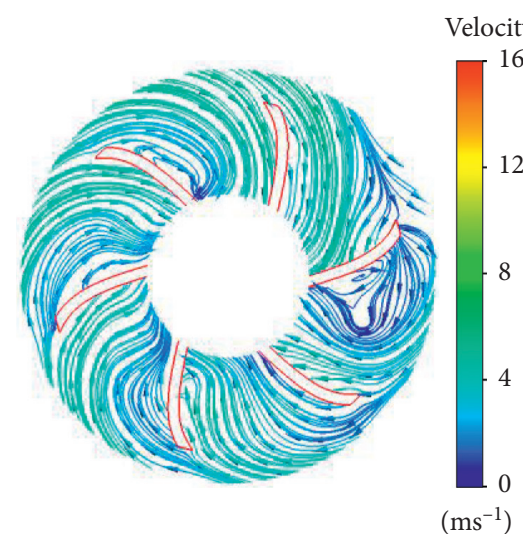

(a)

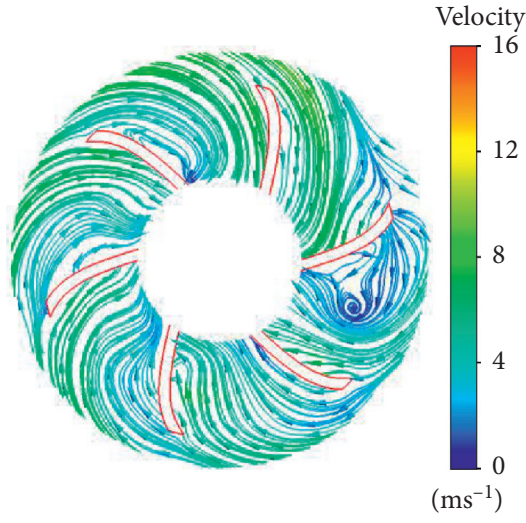

(b)

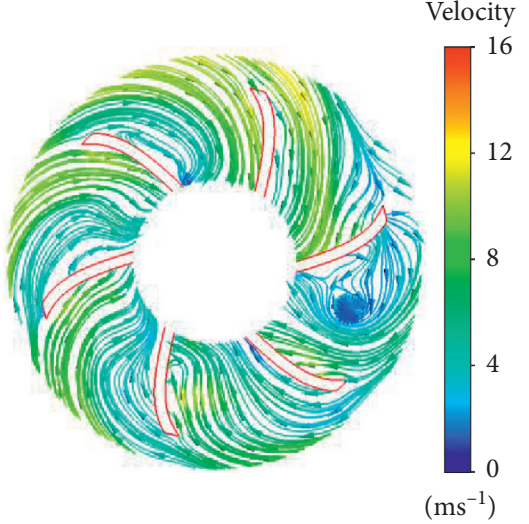

(c)

Figure 5: Velocity streamlines of runner at $z=0$ plane: (a) $1000 \mathrm{r} / \mathrm{min}\left(80 \mathrm{~m}^{3} / \mathrm{h}\right) ;(b) 1500 \mathrm{r} / \mathrm{min}\left(120 \mathrm{~m}^{3} / \mathrm{h}\right) ;$ (c) $2000 \mathrm{r} / \mathrm{min}\left(160 \mathrm{~m}^{3} / \mathrm{h}\right)$.

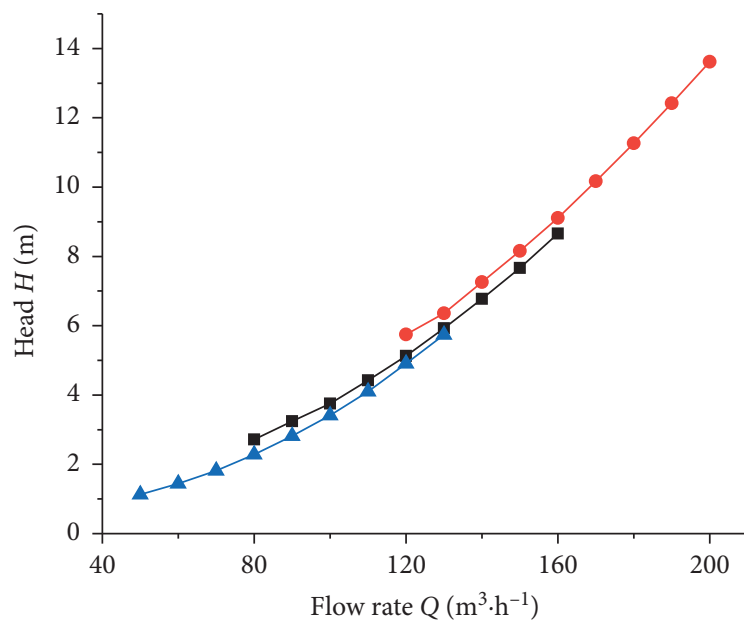

Rotational speed $\left(\mathrm{r} \cdot \mathrm{min}^{-1}\right)$

$\rightarrow 1000 \mathrm{r} / \mathrm{min}$

$\rightarrow 1500 \mathrm{r} / \mathrm{min}$

$\rightarrow-2000 \mathrm{r} / \mathrm{min}$

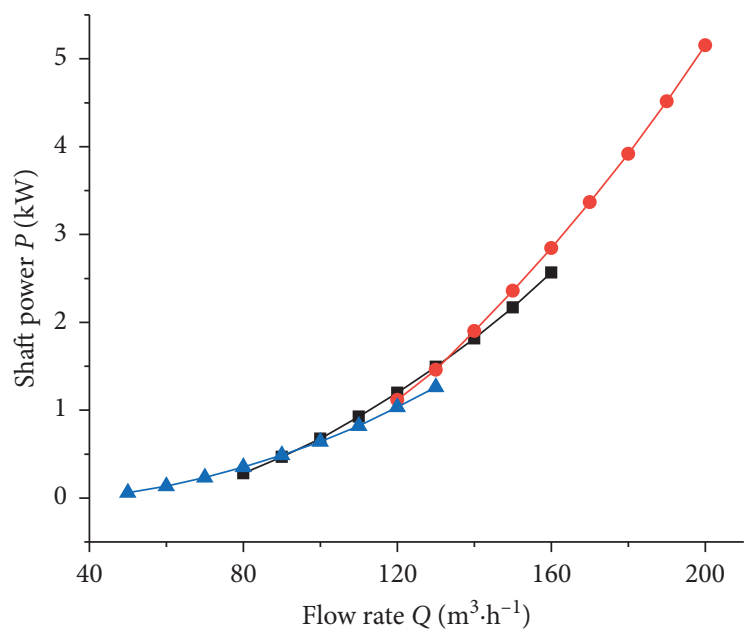

$\longrightarrow 1000 \mathrm{r} / \mathrm{min}$

$\rightarrow 1500 \mathrm{r} / \mathrm{min}$

$\rightarrow 2000 \mathrm{r} / \mathrm{min}$

(a)

(b)

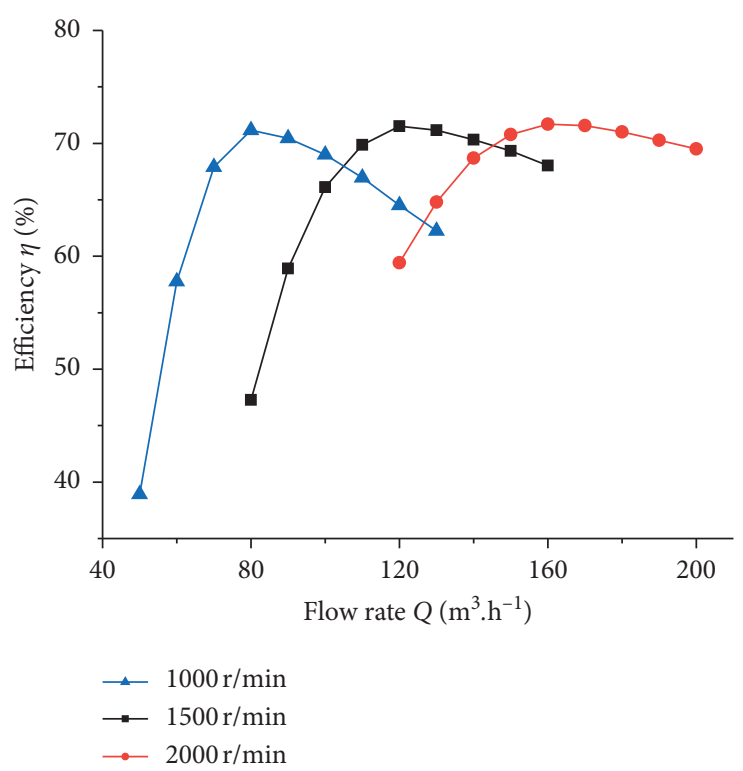

(c)

Figure 6: Performance curves of MF-PAT at different rotational speeds obtained by CFD. 
TABLE 2: BEP parameters by CFD at different rotational speeds.

\begin{tabular}{lccc}
\hline Name & $1000 \mathrm{r} / \mathrm{min}$ & $1500 \mathrm{r} / \mathrm{min}$ & $2000 \mathrm{r} / \mathrm{min}$ \\
\hline$Q\left(\mathrm{~m}^{3} \cdot \mathrm{h}^{-1}\right)$ & 80 & 120 & 160 \\
$H(\mathrm{~m})$ & 2.28 & 5.13 & 9.11 \\
$P(\mathrm{~kW})$ & 0.35 & 1.20 & 2.85 \\
$\mathrm{H}(\%)$ & 71.15 & 71.51 & 71.71 \\
\hline
\end{tabular}

$$
u_{1}=\frac{D_{1} \pi n}{60},
$$

where $D_{1}$ is the outer diameter of impeller and $n$ is the rotational speeds. Impeller's axial velocity $v_{m 1}$ is calculated as follows:

$$
v_{m 1}=\frac{Q \eta_{v}}{F_{1} \phi_{1}},
$$

where $\eta_{v}$ is the volumetric efficiency, $F_{1}$ is the entrance area of impeller, and $\varphi_{1}$ is the blade expelling coefficient. When the geometric parameters of the impeller remain unchanged, the circumferential velocity is proportional to the axial velocity of inlet, which is shown as follows:

$$
\frac{u_{1}}{u_{1}^{\prime}}=\frac{n}{n^{\prime}}=\frac{Q}{Q^{\prime}}=\frac{v_{m 1}}{v_{m 1}^{\prime}}=k .
$$

At the same time, the tangential velocity $v_{u}$ and radial velocity $v_{r}$ of the flow in the volute are in direct proportion to $Q$. When the flow rate increases, the absolute velocity $v$ in the volute changes accordingly, while the direction of $v$ remains unchanged.

From above analysis, it can be concluded that the internal streamline of the impeller only changes in value, and there is little change in direction at different rotational speeds. Moreover, the inlet speed triangle of impeller is drawn under BEPs, as shown in Figure 7.

According to the law of proportionality for centrifugal pump, the various efficiencies are approximately equal when operating under similar conditions. When the rotational speeds change, MF-PAT parameters are shown in the following equations:

$$
\begin{aligned}
& \frac{Q_{1}}{Q_{2}}=\frac{n_{1}}{n_{2}}, \\
& \frac{H_{1}}{H_{2}}=\left(\frac{n_{1}}{n_{2}}\right)^{2}, \\
& \frac{P_{1}}{P_{2}}=\left(\frac{n_{1}}{n_{2}}\right)^{3} .
\end{aligned}
$$

Based on the BEP parameters at the rated speed of $1500 \mathrm{r} / \mathrm{min}$, according to equations (4)-(6), the flow rate, head, and shaft power of BEP were calculated separately at other rotational speeds. Finally, the deviations between theoretical calculation and numerical investigation are listed in Table 3.

In this paper, the turbine efficiency includes hydraulic efficiency, partial volumetric efficiency, and partial mechanical efficiency. However, the volume loss caused by the leakage of the balance hole and the mechanical friction loss of the bearing cannot be reflected in CFD. However, the difference between the calculated results and the theoretical results is very small, indicating the accuracy of CFD.

\section{Experiment}

4.1. Experiment Method. A mixed flow pump with a specific speed of 288 was selected for the experiment to compare with the results acquired from numerical calculations. The head, flow rate, and speed at rated point are $5 \mathrm{~m}, 120 \mathrm{~m}^{3} / \mathrm{s}$, and $1500 \mathrm{r} / \mathrm{min}$, respectively.

Figure 8 shows the schematic diagram of experiment rig. The experiment rig consists of MF-PAT, dynamometer, service pump, underground reservoir, and piping system. The rotation speed of service pump sent liquid with different energies into MF-PAT is controlled by a frequency conversion motor. Then, high-pressure liquid impacted the impeller, causing the impeller to convert the fluid pressure energy into mechanical energy. The regulating valve regulated the flow of MF-PAT. The flowmeter was used to measure the value of the flow entering the turbine. Meanwhile, two piezometric pipes were fixed at MF-PAT's inlet and outlet to measure pressure, respectively, by changing the excitation current of the dynamometer to control the rotation speed of the turbine and measure the output torque. The flow rate, pressure, speed, and torque signals measured on the bench were transmitted to the computer terminal to calculate the head, shaft power, and efficiency of PAT by the test software. Finally, the external characteristic curves were obtained. All experiment instruments were employed after calibration. Figure 9 shows the test of external characteristics. Table 4 shows the parameters of the measurement equipment used in the experiment.

4.2. Experiment Result. Figure 10 shows the external characteristic curves obtained in the experiment, which could present that the trends of the external characteristic curves by experimental are similar to that calculated by CFD. The experimental results also show that the efficiency of the MF-PAT increases firstly and then increases from 1000 to $2000 \mathrm{r} / \mathrm{min}$, while the shaft power and head increase nonlinearly. The BEP also shifts to the large-flow conditions. Table 5 lists the BEP at different rotation speeds in experiment. The deviations from experiment and simulation are less than $5 \%$, which is attributed to leakage loss in the balance hole, the fluid loss along the way, and the mechanical loss in the bearing. Thus, the internal flow field of MF-PAT can be analyzed reasonably through the numerical simulation method.

The experimental results also show the PAT's rotational speeds can be changed to adapt to different flow rates. In other words, the rotational speeds of PAT could be reduced during the dry season and increased during the high water period, which can improve energy recovery efficiency. 


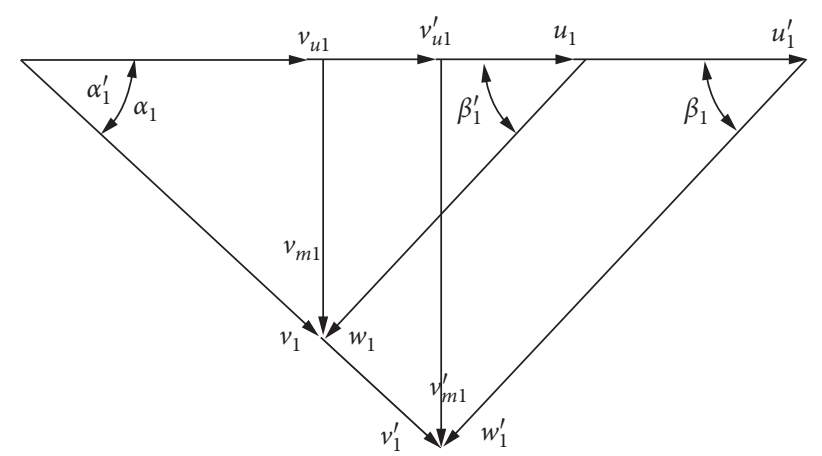

FIGURE 7: Speed triangle change in impeller inlet.

TABLE 3: CFD and theoretical predicted BEP of MF-PAT at different rotational speeds.

\begin{tabular}{|c|c|c|c|c|c|c|c|c|c|c|}
\hline \multirow{2}{*}{$n(\mathrm{r} / \mathrm{min})$} & \multicolumn{3}{|c|}{$Q\left(\mathrm{~m}^{3} \cdot \mathrm{h}^{-1}\right)$} & \multicolumn{3}{|c|}{$H(\mathrm{~m})$} & \multicolumn{3}{|c|}{$P(\mathrm{~kW})$} & \multirow{2}{*}{$\eta(\%)$} \\
\hline & Theory & CFD & $\Delta(\%)$ & Theory & CFD & $\Delta(\%)$ & Theory & CFD & $\Delta(\%)$ & \\
\hline 1000 & 80 & 80 & 0.00 & 2.28 & 2.284 & -0.18 & 0.36 & 0.354 & 0.44 & 71.15 \\
\hline 1500 & 120 & 120 & 0.00 & 5.13 & 5.130 & 0.00 & 1.20 & 1.20 & 0.00 & 71.51 \\
\hline 2000 & 160 & 160 & 0.00 & 9.12 & 9.110 & 0.11 & 2.84 & 2.85 & -0.19 & 71.71 \\
\hline
\end{tabular}

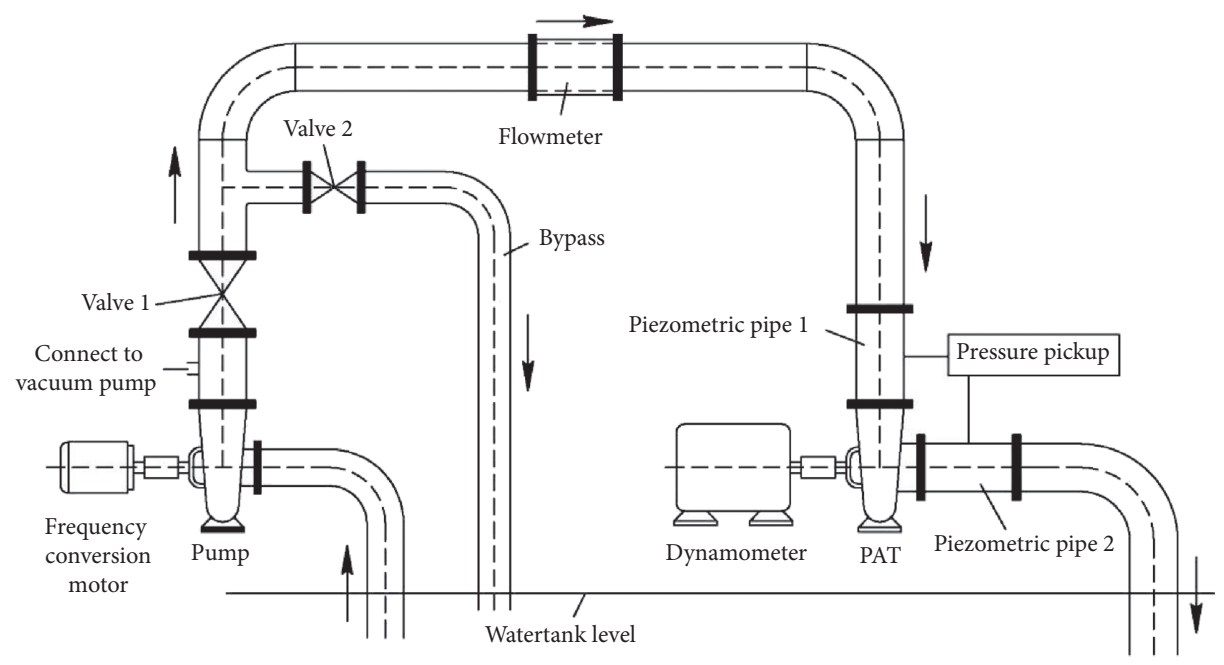

FIgURE 8: Schematic diagram of experiment rig.

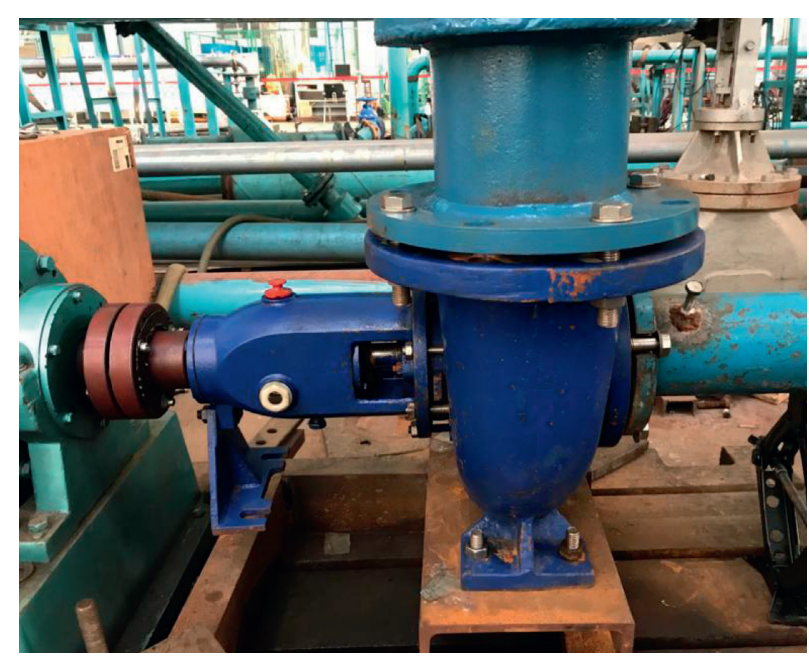

Figure 9: Experimental test of external characteristics. 
TABle 4: Parameters of the measurement equipment.

\begin{tabular}{lcccc}
\hline Equipment name & Equipment model & Measurement content & Range & Accuracy (\%) \\
\hline \multirow{2}{*}{ Dynamometer } & CWF25D & Torque & $0-120 \mathrm{~N} \cdot \mathrm{m}$ & \pm 0.4 \\
& & Rotation speed & $0-8000 \mathrm{rpm}$ & \pm 0.1 \\
\hline \multirow{2}{*}{ Pressure pickup } & WT2000GP6S & Outlet pressure & $-0.3-+0.3 \mathrm{MPa}$ & \pm 0.1 \\
& WT2000GP7S & Inlet pressure & $0-1 \mathrm{MPa}$ & \pm 0.1 \\
\hline Flowmeter & LDBE-150 & Flow & $50-400 \mathrm{~m}^{3} / \mathrm{h}$ & \pm 0.5 \\
\hline
\end{tabular}

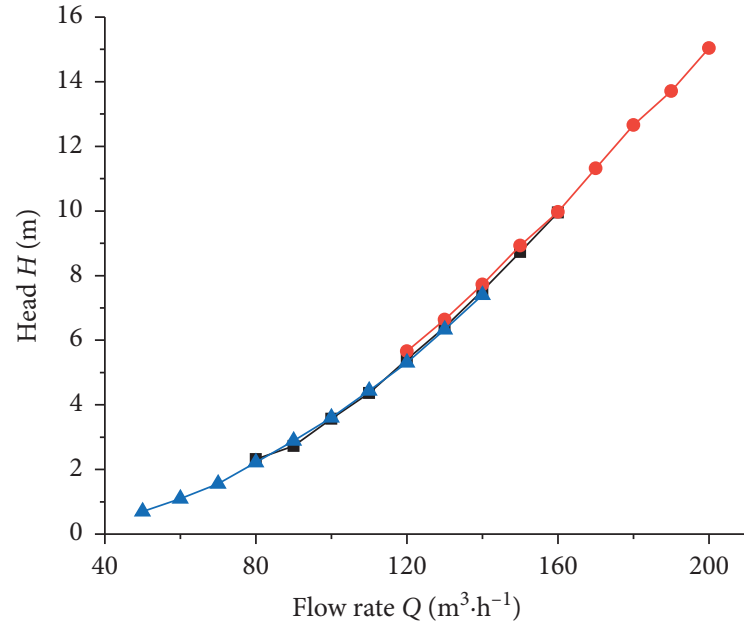

$\simeq 1000 \mathrm{r} / \mathrm{min}$

$\rightarrow 1500 \mathrm{r} / \mathrm{min}$

$\rightarrow 2000 \mathrm{r} / \mathrm{min}$

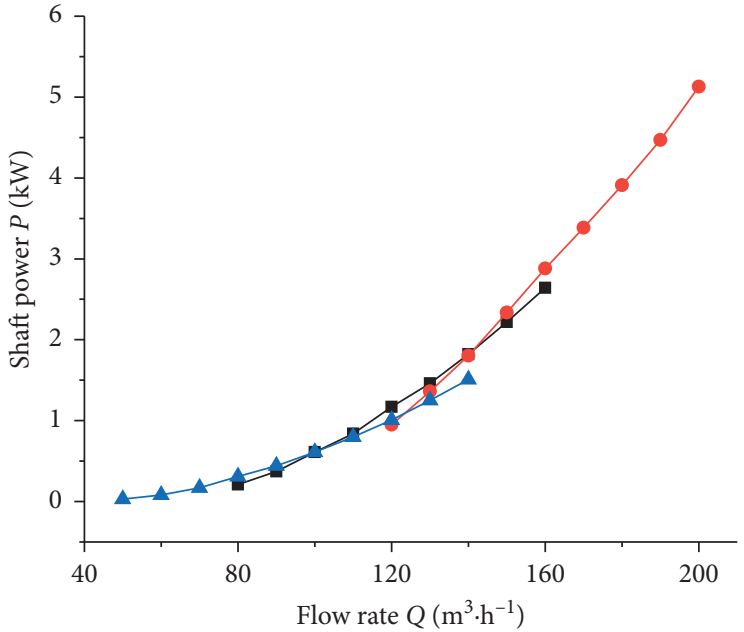

$\simeq 1000 \mathrm{r} / \mathrm{min}$

$\rightarrow 1500 \mathrm{r} / \mathrm{min}$

$\rightarrow 2000 \mathrm{r} / \mathrm{min}$

(a)

(b)

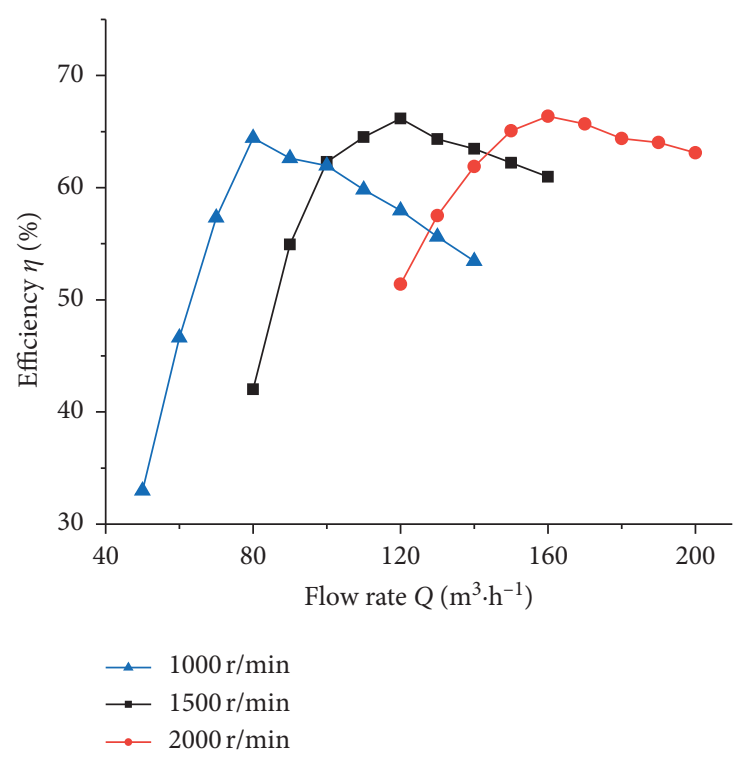

(c)

Figure 10: Performance curves of MF-PAT at different rotational speeds obtained by experiment.

TABLe 5: BEP parameters by experiment at different rotational speeds.

\begin{tabular}{lccc}
\hline Parameters & $1000 \mathrm{r} / \mathrm{min}$ & $1500 \mathrm{r} / \mathrm{min}$ & $2000 \mathrm{r} / \mathrm{min}$ \\
\hline$Q\left(\mathrm{~m}^{3} \cdot \mathrm{h}^{-1}\right)$ & 80 & 120 & 160 \\
$H(\mathrm{~m})$ & 2.22 & 5.41 & 9.97 \\
$P(\mathrm{~kW})$ & 0.31 & 1.17 & 2.88 \\
$\eta(\%)$ & 64.43 & 66.17 & 66.36 \\
\hline
\end{tabular}




\section{Conclusion}

(1) The rotation speed of MF-PAT has a significant impact on its performance through numerical calculations and experiment. With the increase in rotation speed, the turbine is more suitable for large-flow condition, but the maximum efficiency is not improved.

(2) When the rotation speed is changed, the flow rate of the MF-PAT changes positively, the head changes quadratically, and the power changes cubically.

(3) Reasonable adjustment of rotational speeds could improve energy recovery and utilization efficiency. When the hydraulic environment of MF-PAT deviates from the rated working environment, increase in speed is positive to improve the energy recovery efficiency in the high-water period and negative in the dry period.

\section{Data Availability}

The data used to support the findings of this study are included within the article.

\section{Conflicts of Interest}

The authors declare that they have no conflicts of interest.

\section{References}

[1] O. Ellabban, H. Abu-Rub, and F. Blaabjerg, "Renewable energy resources: current status, future prospects and their enabling technology," Renewable and Sustainable Energy Reviews, vol. 39, no. 2, pp. 748-764, 2014.

[2] IRENA, Renewable Energy Statistics 2020, IRENA-International Renewable Energy Agency, Abu Dhabi, United Arab Emirates, 2020.

[3] H. Wang, B. Long, C. Wang, C. Han, and L. Li, "Effects of the impeller blade with a slot structure on the centrifugal pump performance," Energies, vol. 13, no. 7, p. 1628, 2020.

[4] S. Tang, S. Yuan, and Y. Zhu, "Deep learning-based intelligent fault diagnosis methods toward rotating machinery," IEEE Access, vol. 8, pp. 9335-9346, 2020.

[5] S. Tang, S. Yuan, and Y. Zhu, "Data preprocessing techniques in convolutional neural network based on fault diagnosis towards rotating machinery," IEEE Access, vol. 99, p. 1, 2020.

[6] S. Barbarelli, M. Amelio, and G. Florio, "Predictive model estimating the performances of centrifugal pumps used as turbines," Energy, vol. 107, no. 15, pp. 103-121, 2016.

[7] P. Singh, Optimization of Internal Hydraulics and of System Design for Pumps as Turbines with Field Implementation and evaluation, University of Karlsruhe, Karlsruhe, Germany, 2005.

[8] S.-S. Yang, S. Derakhshan, and F.-Y. Kong, "Theoretical, numerical and experimental prediction of pump as turbine performance," Renewable Energy, vol. 48, pp. 507-513, 2012.

[9] P. Singh and F. Nestmann, "An optimization routine on a prediction and selection model for the turbine operation of centrifugal pumps," Experimental Thermal and Fluid Science, vol. 34, no. 2, pp. 152-164, 2010.

[10] S. Derakhshan and A. Nourbakhsh, "Theoretical, numerical and experimental investigation of centrifugal pumps in reverse operation," Experimental Thermal and Fluid Science, vol. 32, no. 8, pp. 1620-1627, 2008.

[11] T. Wang, F. Kong, B. Xia, Y. Bai, and C. Wang, "The method for determining blade inlet angle of special impeller using in turbine mode of centrifugal pump as turbine," Renewable Energy, vol. 109, pp. 518-528, 2017.

[12] S. Derakhshan and A. Nourbakhsh, "Experimental study of characteristic curves of centrifugal pumps working as turbines in different specific speeds," Experimental Thermal and Fluid Science, vol. 32, no. 3, pp. 800-807, 2008.

[13] S. Abazariyan, R. Rafee, and S. Derakhshan, "Experimental study of viscosity effects on a pump as turbine performance," Renewable Energy, vol. 127, pp. 539-547, 2018.

[14] S.-S. Yang, F.-Y. Kong, W.-M. Jiang, and X.-Y. Qu, "Effects of impeller trimming influencing pump as turbine," Computers \& Fluids, vol. 67, pp. 72-78, 2012.

[15] S. S. Yang, H. L. Liu, F. Y. Kong, B. Xia, and L. W. Tan, "Effects of the radial gap between impeller tips and volute tongue influencing the performance and pressure pulsations of pump as turbine," Journal of Fluids Engineering, vol. 136, no. 5, Article ID 054501, 2014.

[16] S. Barbarelli, G. Florio, M. Amelio, N. M. Scornaienchi, A. Cutrupi, and G. Lo Zupone, "Design procedure of an innovative turbine with rotors rotating in opposite directions for the exploitation of the tidal currents," Energy, vol. 77, pp. 254-264, 2014.

[17] X. Q. Tu, "Selection method of energy recovery hydraulic turbine," General Machinery, vol. 5, pp. 40-42, 2010.

[18] L. Shi, W. Zhang, H. Jiao et al., "Numerical simulation and experimental study on the comparison of the hydraulic characteristics of an axial-flow pump and a full tubular pump," Renewable Energy, vol. 153, pp. 1455-1464, 2020.

[19] L. Zhou, W. D. Shi, L. Bai, W. G. Lu, and W. Li, "Numerical calculation and experimental study of axial force in a deepwell centrifugal pump," Investigación Aplicada Latinoamericana, vol. 44, no. 1, pp. 105-110, 2014.

[20] M. A. Demaria, M. Casto, M. O’Connell, R. P. Johnson, and M. Rosenzweig, "Application of high-voltage frequency conversion device in condensate pump energy saving reformation of thermal power plant," Heb Electric Power, vol. 12, no. 4, pp. $1650-1658,2010$.

[21] J. Huang, L. Zou, Z. Li, X. Wang, and Y. Wang, "Development and performance comparison of valveless piezoelectric pumps with asymmetrical channels," Sensors and Actuators A Physical, vol. 314, Article ID 112241, 2020.

[22] Y. Yang, L. Zhou, W. Shi, Z. He, and Y. Xiao, "Interstage difference of pressure pulsation in a three-stage electrical submersible pump," Journal of Petroleum Ence and Engineering, vol. 196, Article ID 107653, 2020.

[23] X. He, Y. Zhang, C. Wang et al., "Influence of critical wall roughness on the performance of double-channel sewage pump," Energies, vol. 13, no. 2, p. 464, 2020. 